\title{
Reconfigurable Acoustic Metagrating for High-Efficiency Anomalous Reflection
}

\author{
Yan Kei Chiang, ${ }^{1, *}$ Sebastian Oberst $\odot,{ }^{1,2}$ Anton Melnikov $\odot,{ }^{3,4}$ Li Quan, ${ }^{5}$ Steffen Marburg, ${ }^{3}$ \\ Andrea Alù, ${ }^{5,6}$ and David A. Powell ${ }^{1,2, \dagger}$ \\ ${ }^{1}$ School of Engineering and Information Technology, University of New South Wales, Canberra, Australia \\ ${ }^{2}$ Centre for Audio, Acoustics and Vibration, University of Technology, Sydney, Australia \\ ${ }^{3}$ Vibroacoustics of Vehicles and Machines, Technical University, Munich, Germany \\ ${ }^{4}$ Fraunhofer Institute for Photonic Microsystems, Dresden, Germany \\ ${ }^{5}$ Department of Electrical and Computer Engineering, The University of Texas at Austin, Austin, Texas 78712, \\ USA \\ ${ }^{6}$ Photonics Initiative, Advanced Science Research Center, City University of New York, New York, New York, USA
}

(Received 30 October 2019; revised manuscript received 14 April 2020; accepted 26 May 2020; published 29 June 2020)

\begin{abstract}
Metagratings are a recently proposed class of metasurfaces for efficient manipulation of an impinging wavefront within a subwavelength layer. They avoid the requirement for fine discretization of gradient metasurfaces, and overcome their inherent limitations in efficiency. Here, we demonstrate experimentally the functioning principle of a reconfigurable acoustic metagrating for anomalous reflection with high efficiency, using coarse geometric design features. It is formed by a periodic array of C-shaped meta-atoms, which exhibit large Willis coupling, resulting in a controlled level of asymmetry in their scattering pattern. Our results reveal that the proposed acoustic metagrating can reroute an incident wave towards a large angle, beyond the limitations of gradient-phase approaches with nearly unitary reflection efficiency. The proposed designs offer a highly efficient tunable platform to control steering angle and operating frequency. In our experiments, an acoustic wave is successfully steered to the desired reflection direction by finite metagratings, demonstrating reconfigurability in angle and operating frequency.
\end{abstract}

DOI: 10.1103/PhysRevApplied.13.064067

\section{INTRODUCTION}

Artificial acoustic metamaterials are rationally designed structures with subwavelength characteristics, with unprecedented properties compared to natural materials. In particular, the emergence of acoustic metasurfaces has offered opportunities for acoustic wavefront manipulation, facilitating, e.g., pioneering the design of sound diffusers [1], acoustic holography devices [2-4], and ultrasonic imaging technologies [5]. Acoustic metasurfaces have the capacity to steer reflected or transmitted wavefronts by carefully engineering a spatial gradient of phase, in accordance with generalized Snell's law [6,7]. Phase gradients can be realized by simple passive devices using either labyrinthine structure, which allow the sound wave to accumulate large phase changes [8-10], or Helmholtz resonators, which modulate the phase shift based on their resonant features [11,12]. However, it has been demonstrated that the wavefront steering of phase-gradient metasurfaces can be inefficient due to the impedance mismatch between the incident wave and waves scattered in the

\footnotetext{
*y.chiang@adfa.edu.au

†david.powell@adfa.edu.au
}

desired direction $[13,14]$. For a phase-gradient metasurface performing beam steering at a large angle, this mismatch leads to significant scattering of energy into spurious directions.

For transmissive gradient metasurfaces, the efficiency of wavefront manipulation for large steering angles can be greatly improved using bianisotropy [15]. However, the large variation in surface impedance requires highresolution discretization, and subsequently enhanced fabrication complexity. Furthermore, this approach does not work for reflection, and it becomes necessary to consider highly nonlocal responses $[16,17]$. On the other hand, metagratings are periodic arrays of discrete elements with engineered scattering properties, which have been shown to overcome these limitations. In electrodynamics, metagratings based on asymmetric bianisotropic scatterers have been shown to steer propagating waves with near unitary efficiency [18-21].

Bianisotropy in electromagnetics has its analog in acoustics, known as Willis coupling [22], which represents the cross-coupling between strain and velocity responses. It provides an additional degree of freedom to control an acoustic beam. Willis coupling only occurs in asymmetric structures, and it has been theoretically and 
experimentally demonstrated that Willis coupling must be considered when determining the effective material properties of asymmetric metamaterials [23]. Willis coupling leads to different values of the complex reflection coefficient for opposite propagation directions, as was demonstrated by Liu et al. for flexural wave propagation on both beamlike and platelike structures [24]. Quan et al. theoretically derived the general bounds on Willis coupling in passive scatterers, and showed that such scatterers can be used for anomalous reflection (i.e., reflection at a nonspecular angle) at large angles with full efficiency [25]. The performance of acoustic metagratings incorporating Willis coupling was demonstrated experimentally for both refracted and reflected waves by Craig et al. [26]. However, their operation was restricted to relatively low angles of incidence and refraction, of up to $45^{\circ}$. To overcome this restriction, Torrent, $\mathrm{Ni}$ et al., and Hou et al. proposed another metagrating structure by etching several straight-walled grooves on a hard surface for larger steering angles [27-29]. They demonstrated that their proposed structure could achieve high reflected or refracted beam steering efficiency. In these works, metagrating structures were designed and machined for several operating scenarios with a fixed angle and fixed operating frequency. Thus a more versatile metagrating structure, which can be configured to achieve high efficiency for a wide range of angles and frequencies, remains to be demonstrated.
In this work, we propose an acoustic metagrating with reconfigurable wavefront manipulation, based on an array of C-shaped meta-atoms (individual structured elements forming the metamaterial). We take advantage of our recent study which experimentally evidenced maximal Willis coupling in easy to fabricate $\mathrm{C}$-shaped meta-atoms [30]. We now apply this geometry as the building block of metagrating designs, demonstrating that the same inclusion can be used to implement different metagratings, allowing variation of the steering angle and operating frequency. Our results show that this technique can support highly efficient beam steering for anomalous reflection into large angles.

\section{DEMONSTRATION OF METAGRATING FOR EXTREME REFLECTION ANGLES}

\section{A. Metagrating design}

Our metagrating designs consist of periodically arranged C-shaped meta-atoms separated from a hard wall by a distance $H$. Each meta-atom is a hollow cylinder with outer and inner radius $r_{a}$ and $r_{i}$, respectively, and an aperture of width $w_{o}$. The unit cell of the metagrating is shown in Fig. 1(a), and a complete metagrating is illustrated in Fig. 1(b). Initially we model the metagrating as having infinite length. In addition to the meta-atom geometry, the (a)

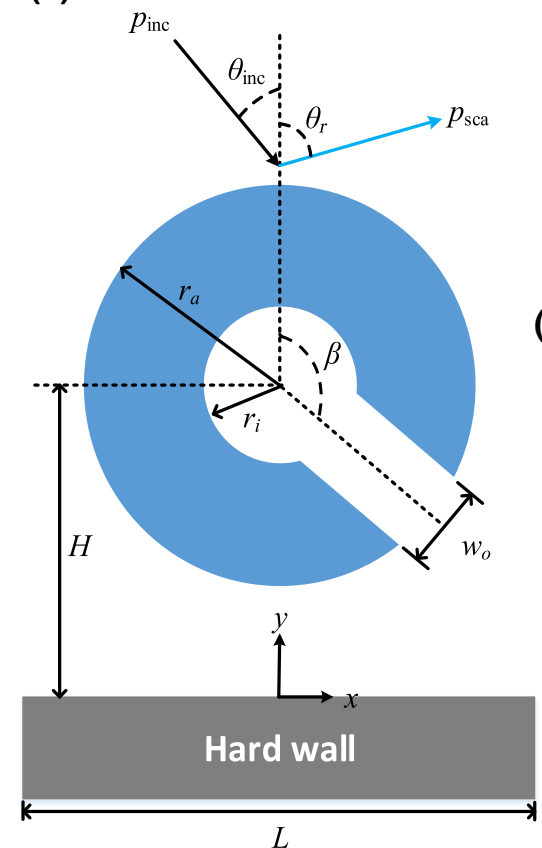

(b)

(c)

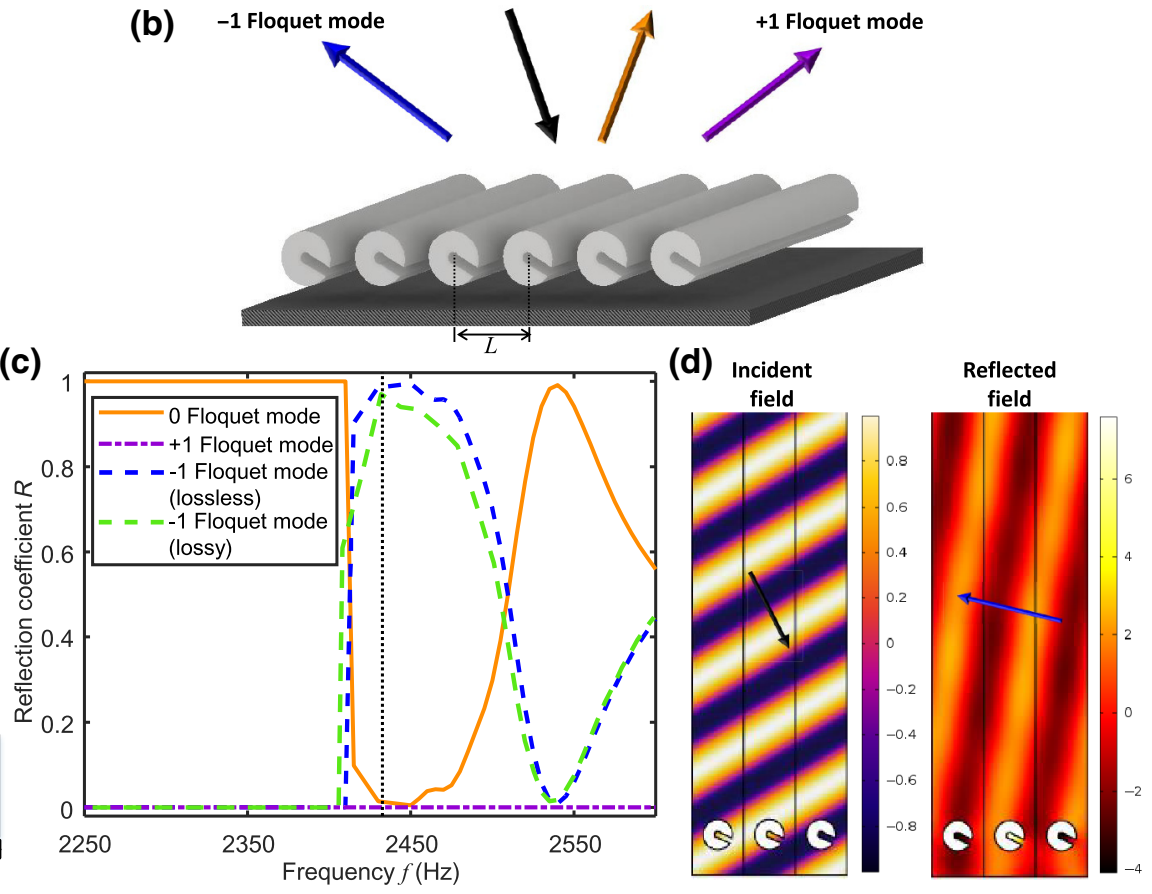

0 Floquet mode

FIG. 1. Design of reconfigurable acoustic metagrating. (a) Two-dimensional configuration of the C-shaped meta-atom and hard wall forming the unit cell of the proposed metagrating. (b) Schematic of metagrating indicating the incident wave and three propagating Floquet modes. (c) Reflection coefficient for 0 (solid line), -1 (dashed line), and +1 (dash-dotted line) Floquet modes of our designed metagrating. (d) Real part of incident and reflected pressure fields ( -1 Floquet mode) at $f=2430 \mathrm{~Hz}$. 
twist angle $\beta$ and the distance $H$ to the hard wall must be chosen carefully to achieve maximum efficiency.

A plane wave impinging on the metagrating may reflect energy via multiple Floquet modes (i.e., the structure can diffract energy into several diffraction orders), with the number of modes determined by the ratio of the metagrating period $L$ to the acoustic wavelength $\lambda$. Careful choice of the meta-atom geometry and location can in principle result in all energy being diffracted into a single Floquet mode. For the range of $L$ and $\lambda$, that we consider, three propagating Floquet modes need to be taken into account $(-1,0$, and +1$)$. The period $L$ of the metagrating is chosen such that the -1 Floquet mode is reflected at the desired angle $\theta_{-1}$, based on Bragg's condition

$$
L=\lambda_{0} /\left(\sin \theta_{\text {inc }}-\sin \theta_{-1}\right),
$$

where $\lambda_{0}$ denotes the operating wavelength in free space and $\theta_{\text {inc }}$ is the angle of incidence. $\theta_{\text {inc }}$ and $\theta_{-1}$ are defined relative to the surface normal of the metagrating. To establish a high-efficiency metagrating for anomalous reflection three design criteria are required: (1) a high impedance mismatch between the incident wave and metagrating such that high reflection is achieved; (2) a scatterer with large Willis coupling to provide asymmetric scattering; (3) its Willis properties should be adjustable such that the amplitude of the asymmetric reflection can be controlled. A hard wall is adopted in our proposed design to ensure high reflection efficiency. On its own, the wall would cause unwanted reflection in the specular direction. To precisely cancel this reflection, the interference between the reflected wave from the wall and the specular scattered wave from the meta-atoms is carefully adjusted by tuning the distance $H$ between the scatterers and the hard wall. In Ref. [18] it was shown how $H$ can be chosen for electromagnetic metagratings, based on the operating frequency, polarizability of the meta-atom, and angles of incidence and reflection. This result can be adapted to acoustic metagratings, however it neglects near-field effects, which occur when a finite-sized scatterer is placed close to a surface, thus it provides only an initial estimate of the optimal position. Although our optimal value of $H$ differs from this estimate, the basic conclusion remains that the thickness of the metagrating cannot be arbitrarily smaller than the wavelength.

After choosing $L$ and the initial estimate of $H$, we design a C-shaped meta-atom with large Willis coupling at its resonance. The operating frequency of the metagrating is associated with the resonance of the scatterer. Furthermore, our C-shaped meta-atom can be twisted about its origin to simultaneous change the longitudinal and transverse components of Willis coupling. We design the meta-atom to have zero scattering into +1 Floquet mode and with scattering in the specular direction ( 0 Floquet mode) to cancel the reflection of the incident wave from the hard wall by tuning the twist angle $\beta$ and distance $H$ from the hard wall. The effects of the geometric parameters and the dependence of the beam steering on the Willis coupling is discussed in Sec. 4 within the Supplemental Material [32]. In this way, the meta-atoms and hard wall are combined into a metagrating, which performs anomalous reflection in the direction of the -1 Floquet mode with near unitary efficiency.

To demonstrate the beam-reflection efficiency at large angles, we design a metagrating, which can efficiently reroute an oblique incident wave with $\theta_{\text {inc }}=30^{\circ}$ to an extreme angle $\theta_{-1}=-80^{\circ}$ at $f_{0}=2430 \mathrm{~Hz}$. We choose the $\mathrm{C}$-shaped meta-atoms with dimensions $r_{a}=27 \mathrm{~mm}$, $r_{i}=6 \mathrm{~mm}$, and $w_{o}=10 \mathrm{~mm}$, since those designs have demonstrated large Willis coupling at their resonance, of about $86 \%$ of the theoretical maximum [30]. The twist angle $\beta=115^{\circ}$ and the distance $H=0.0735 \mathrm{~m}$ from the hard wall are chosen empirically by parametric sweeps based on the initial estimates obtained from the aforementioned design criteria. The corresponding polarizability tensor of the meta-atoms is shown in Fig. S1 within the Supplemental Material [32]. The fundamental resonance of an individual meta-atom is $2200 \mathrm{~Hz}$, thus our metagrating operates above this resonance (Fig. S1 within the Supplemental Material [32]). The period of the metagrating is set as $L=0.0951 \mathrm{~m}$ to satisfy Eq. (1) at $f_{0}=2430$ $\mathrm{Hz}$. The full methodology for numerically characterizing the infinite length metagrating is outlined in Sec. 6 within the Supplemental Material [32].

Figure 1(c) shows the numerically calculated reflection into each Floquet mode as a function of frequency. At $f=2430 \mathrm{~Hz}$ the reflection coefficient expressed by Eq. (A4) for the -1 Floquet mode is 0.99 and that for the 0 Floquet mode is close to zero for the lossless case, which indicates that the incident wave is reflected almost completely into the -1 Floquet mode. The simulated pressure distribution illustrated in Fig. 1(d) shows that the metagrating can successfully reroute an obliquely incident wave towards an extreme steering angle $\theta_{-1}=-80^{\circ}$ with negligible scattering in the specular direction. However, the promising beam-steering performance of the metagrating might be degraded if the effect of thermoviscous losses is dominant. To investigate this effect, we establish another finite-element model with thermoviscous losses, with the resulting reflection spectrum indicated by the green dashed line in Fig. 1(c). The thermoviscous losses leads to 3\% reduction in the reflection coefficient at $f=2430 \mathrm{~Hz}$. Furthermore, the reflection spectrum of the lossy metagrating is slightly shifted to lower frequency due to the downshifted Willis coupling reported in Ref. [30]. These results demonstrate that the effect of thermoviscous losses on the performance of our metagrating formed by $\mathrm{C}$-shaped meta-atoms is not significant.

To experimentally validate our metagrating, the performance of the finite-length metagrating is measured 


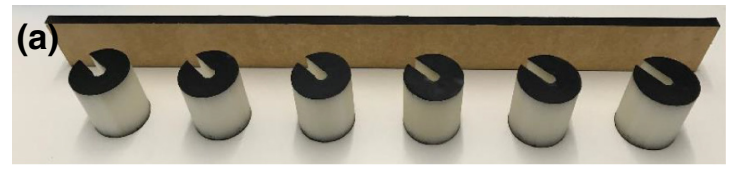

(c)
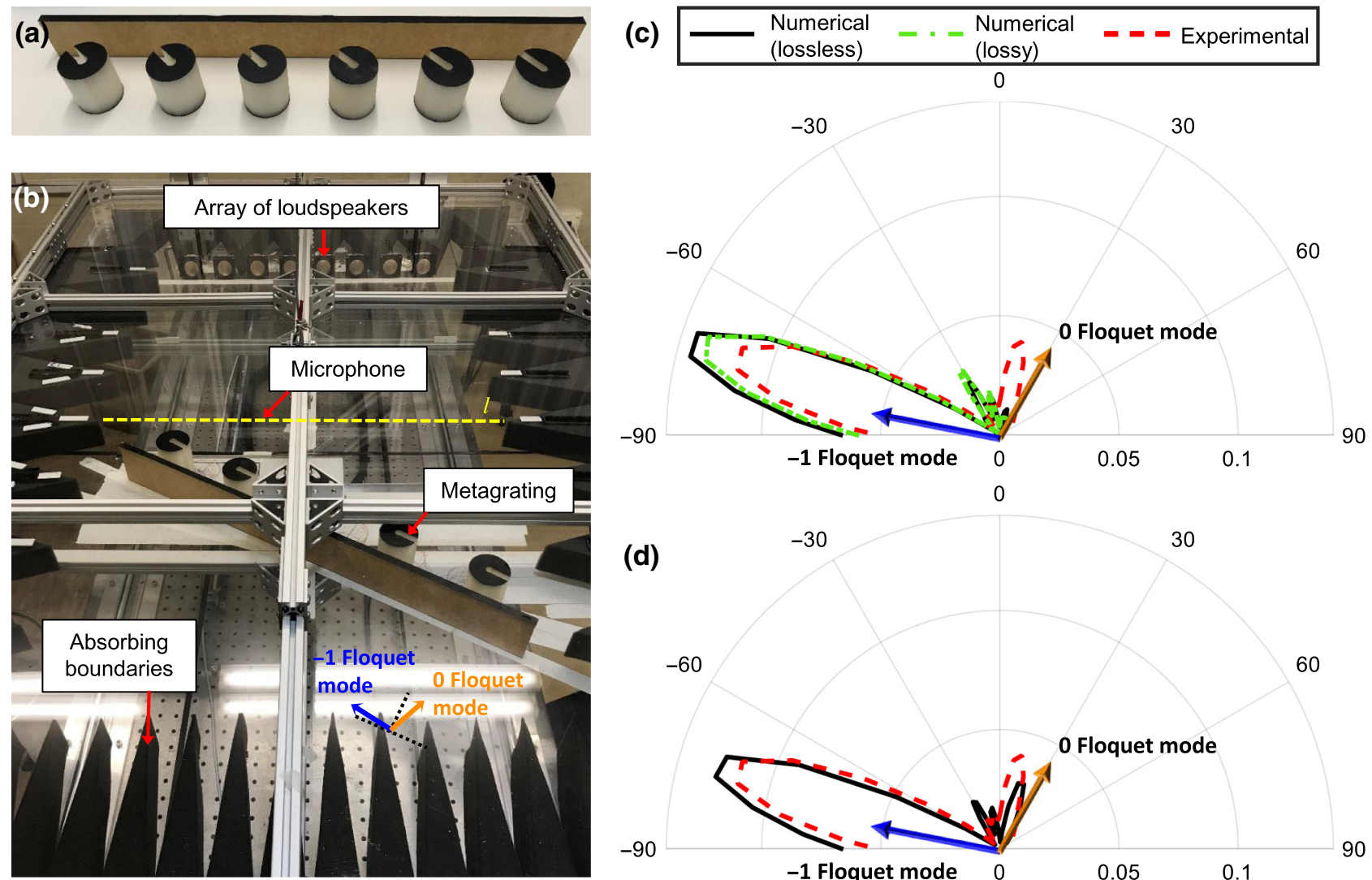

(d)

$-90$

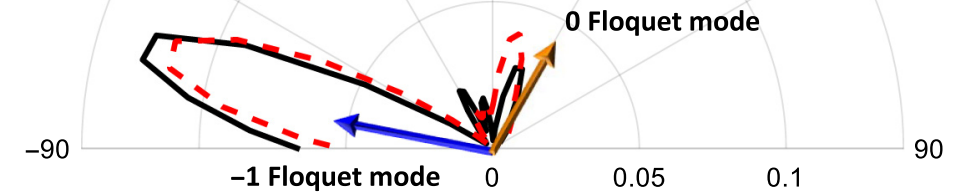

0 Floquet mode

FIG. 2. Experimental demonstration of large-angle anomalous reflection. (a) 3D-printed C-shaped meta-atoms forming reconfigurable acoustic metagratings. (b) Experimental setup using a parallel plate waveguide apparatus, cf. Ref. [31]. The scattered acoustic pressure is measured with a microphone scanned along the yellow dashed line. (c) Polar plot of acoustic pressure comparing the experimentally measured (dashed line) and numerically predicted (solid line) scattering pattern at $f=2430 \mathrm{~Hz}$. (d) Comparison of experimental scattering pattern with numerical results obtained by adjusting period $L$ to $0.093 \mathrm{~m}$ to account for sample misalignment.

experimentally and compared with numerical predictions shown in Fig. 2. Six meta-atoms are 3D printed via fused deposition modeling with polylactic acid (PLA), and a 10mm-thick acrylic plate is used as the acoustically rigid backing wall of the metagrating. As shown in Fig. 2(a), the metagrating is placed into a two-dimensional parallel-plate waveguide with absorbing boundaries. The experimental setup consists of an array of loudspeakers to generate a plane wave and a microphone mounted on a belt system to scan the acoustic pressure within the $x-y$ plane [Fig. 2(b)]. The signal generation and acquisition, and the movement of the microphone are controlled by microcontroller boards with audio and motor control peripherals [31]. To apply the obliquely incident wave, the metagrating is oriented with its surface normal at an angle $\theta_{\text {inc }}=30^{\circ}$ relative to the direction of incidence of the fixed sound source.

\section{B. Experimental verification}

The performance of the metagratings is quantified from experimental and numerical results, using the procedure outlined in Sec. 7 within the Supplemental Material [32].
The incident field is obtained by measuring the empty waveguide, by subtracting it from the total field in the presence of the metagrating, to obtain the scattered field. The scattered pressure is measured along a line in front of the metasurface, and converted to an angular scattered pressure using Eq. (B1). This is used to obtain the angular scattering pattern $\sigma(\theta)$, which is normalized to the total scattered power by Eq. (B3).

Figure 2(c) shows the angular scattering pattern $\sigma(\theta)$ at $f=2430 \mathrm{~Hz}$. The experimentally measured main lobe is shown by the dashed line, with its angle of peak scattering being shifted to $-75^{\circ}$; some loss of acoustic energy is observed, due to scattering into undesired directions. We attribute these effects primarily to the finite width of the metagrating, which is limited by the size of our experimental setup, and which leads to a narrow effective aperture for such large reflection angles. As the aperture size of the metagrating is reduced, the cancellation of unwanted Floquet modes becomes poorer, and the efficiency is reduced. This leads to a trade-off between the beam-steering efficiency and the size of the metagrating, which is especially serious at extreme reflection angles. It should be noted that 
the cancellation of the unwanted diffraction orders can be exactly realized only in infinite metagratings. Similar performance can be observed from the numerical results for the lossless finite-length metagrating, as indicated by the solid line in Fig. 2(c).

The experimentally measured and numerically predicted efficiency values are 0.67 and 0.76 , respectively. To investigate the cause of this difference, the thermoviscous losses are also considered in our finite-element model. A similar angular scattering pattern is shown by comparing the numerical prediction of the lossless (solid line) and lossy cases (dash-dotted line). As in the infinite case, the effect of thermoviscous losses is relatively insignificant, leading to a reduction of $2.6 \%$ in the efficiency of the finitelength metagrating. Therefore, the difference between the numerical and experimental results can be attributed to the imperfect placement of the meta-atoms instead of the effect of thermoviscous losses. In Fig. 2(d) we compare the experimental scattering pattern with lossless numerical results calculated with $L$ reduced to $0.093 \mathrm{~m}$. This adjustment leads to better agreement in the peak scattering, and it predicts the side lobe, which appears at $15^{\circ}$. The remaining disagreement may be attributed to other sources of misalignment in the experiment. The beamsteering performance of our metagrating greatly depends on the Willis properties of the scatterer, which are determined by its geometric parameters. Reduction in efficiency and shift of reflection angle as well as the operating frequency would be induced by the misalignment of a twist angle $\beta$ and an inaccurate dimensions of the samples,
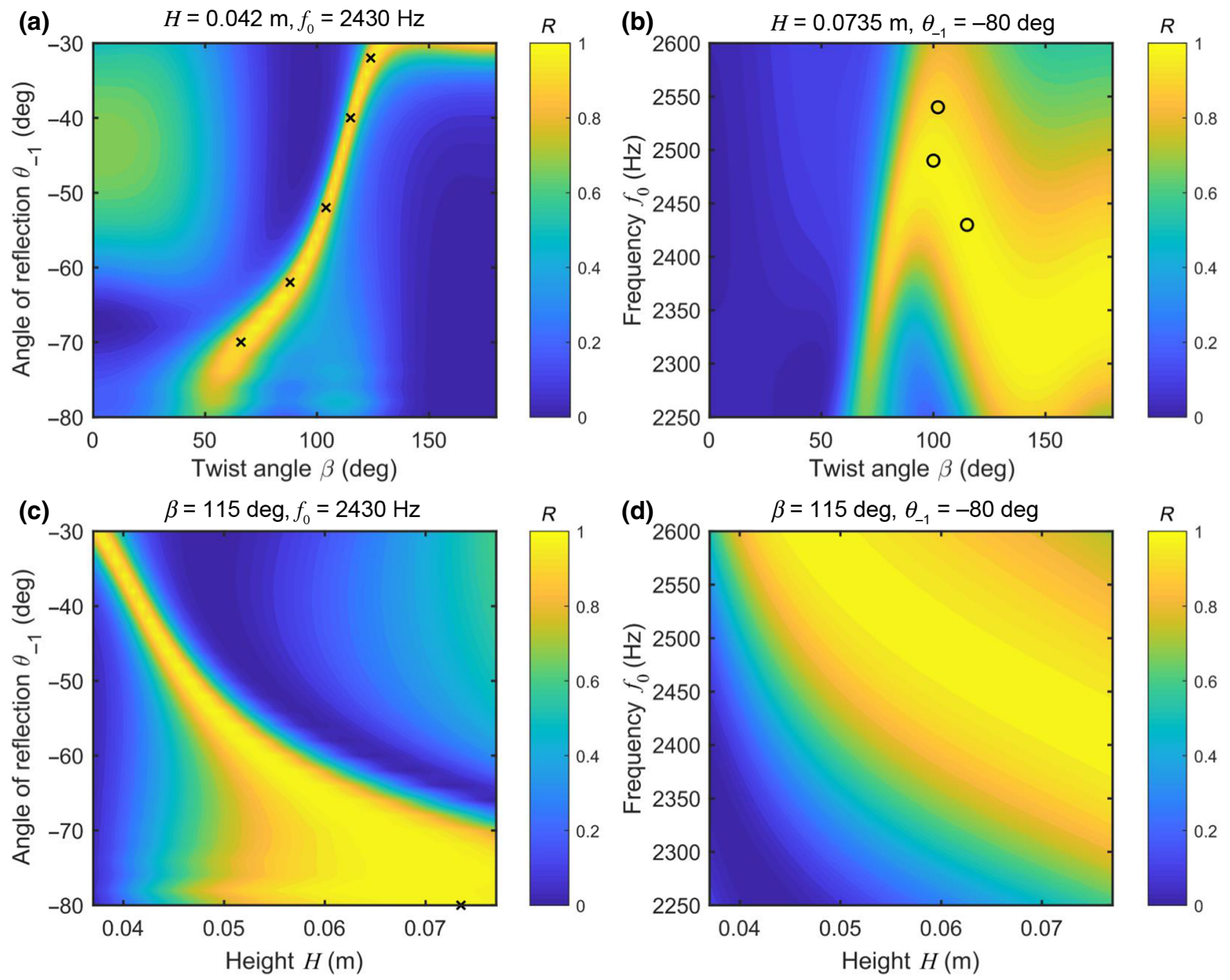

FIG. 3. Numerical demonstration of reconfigurability of our metagrating. (a) Change of reflection angle $\theta_{-1}$ by varying twist angle $\beta$, with $f_{0}=2430 \mathrm{~Hz}$ and $H=0.042 \mathrm{~m}$; (b) change of operating frequency $f_{0}$ by varying angle $\beta$, for reflection angle $\theta_{-1}=-80^{\circ}$ with $H=0.0735 \mathrm{~m}$; (c) change of reflection angle $\theta_{-1}$ by varying distance $H$, with $f_{0}=2430 \mathrm{~Hz}$; and (d) change of operating frequency $f_{0}$ by varying $H$, for reflection angle $\theta_{-1}=-80^{\circ}$. For (c) and (d), $\beta$ is fixed as $115^{\circ}$. Cross and circle markers in (a), (b), and (c) indicate the parameters chosen for experimental validation. In all cases $\theta_{\text {inc }}=30^{\circ}$, and the period $L$ is adjusted along with $\beta$ or $H$ to be consistent with $f_{0}$ and $\theta_{-1}$. 
i.e., $r_{a}, r_{i}$, and $w_{o}$. The performance dependency on the geometric parameters are discussed in Sec. 4 within the Supplemental Material [32].

While the adjustment of $L$ achieves good agreement between the experimental and numerical results for a metagrating with six meta-atoms, these results differ from the numerical results for an infinitely long metagrating. In particular, the main lobe of the finite structure occurs at $-75^{\circ}$ with an efficiency of 0.7 , whereas Eq. (1) predicts that the -1 Floquet harmonic will propagate at $\theta_{-1}=-80^{\circ}$, and Fig. 1(c) predicts an efficiency of 0.99 . To confirm that the shift in angle and reduction in efficiency are due to the finite-length metagrating, we perform a series of numerical simulations with increasing number of meta-atoms $N$. The method and numerical results are detailed in Sec. 2 within the Supplemental Material [32], and show that increasing $N$, to 25 leads to an efficiency of 0.93 at $\theta_{\mathrm{r}}=-79^{\circ}$.

\section{RECONFIGURABILITY OF THE METAGRATING}

\section{A. Control of reflection angle and operating frequency}

To demonstrate the reconfigurablility of our metagrating, we vary $\beta$, the angle of each meta-atom's aperture relative to the surface normal, and $H$, the separation between the meta-atom and the hard wall, cf. Fig. 3(a). By varying these two parameters, we show that a $\mathrm{C}$-shaped meta-atom of fixed geometry can perform anomalous reflection into a range of different angles, and with a controllable operating frequency. Note that we simultaneously vary the period $L$ according to Eq. (1) to remain consistent with the operating frequency and reflection angle. We start by assuming an infinite-length metagrating, and quantify performance as the fraction of energy reflected into the -1 Floquet mode, cf. Eq. (A4).

The numerical results presented in Fig. 3(a) show how changing the rotation angle $\beta$ of each meta-atom is able to tune the angle of refraction, for a fixed operating frequency of $2430 \mathrm{~Hz}$. Alternatively, as shown in Fig. 3(b), by modifying $\beta$ the operating frequency can be varied, while keeping the refraction angle constant at $-80^{\circ}$. We note that the difference between these two cases is determined by the choice of $L$. In Figs. 3(c) and 3(d) we show results for an alternative reconfiguration mechanism, where $H$ is varied to change $f_{0}$ and $\theta_{-1}$, respectively.

Figure 3(a) shows that the metagrating operating at $f_{0}=2430 \mathrm{~Hz}$ with $H=0.042 \mathrm{~m}$ can achieve anomalous reflection with high efficiency, i.e., $R>0.9$, tuned over a wide range of reflection angles from $-30^{\circ}$ to $-72^{\circ}$ by adjusting $\beta$. The optimal operating frequency at an extreme steering angle $\theta_{-1}=-80^{\circ}$ can also be tuned by adjusting $\beta$. The effect of this rotation can be understood via its influence on the effective polarizability of the meta-atom. Figure 3(d) demonstrates that for larger $H$ the operating frequency is reduced due to the increase in accumulated phase between the meta-atom and the hard wall, meaning that the cancellation of specular reflections occurs at a lower frequency. Figure 3(c) shows that a larger separation $H$ leads to efficient reflections at larger angles.

\section{B. Experimental demonstration of reconfigurability}

To experimentally demonstrate the reconfigurability of the metagrating, eight designs are chosen, indicated by the cross $(x)$ and circle $(\circ)$ shown in Fig. 3. The full design parameters of each metagrating are listed in Tables I and II in Sec. 8 within the Supplemental Material [32]. The same markers are used in Figs. 4(a) and 4(b) to represent
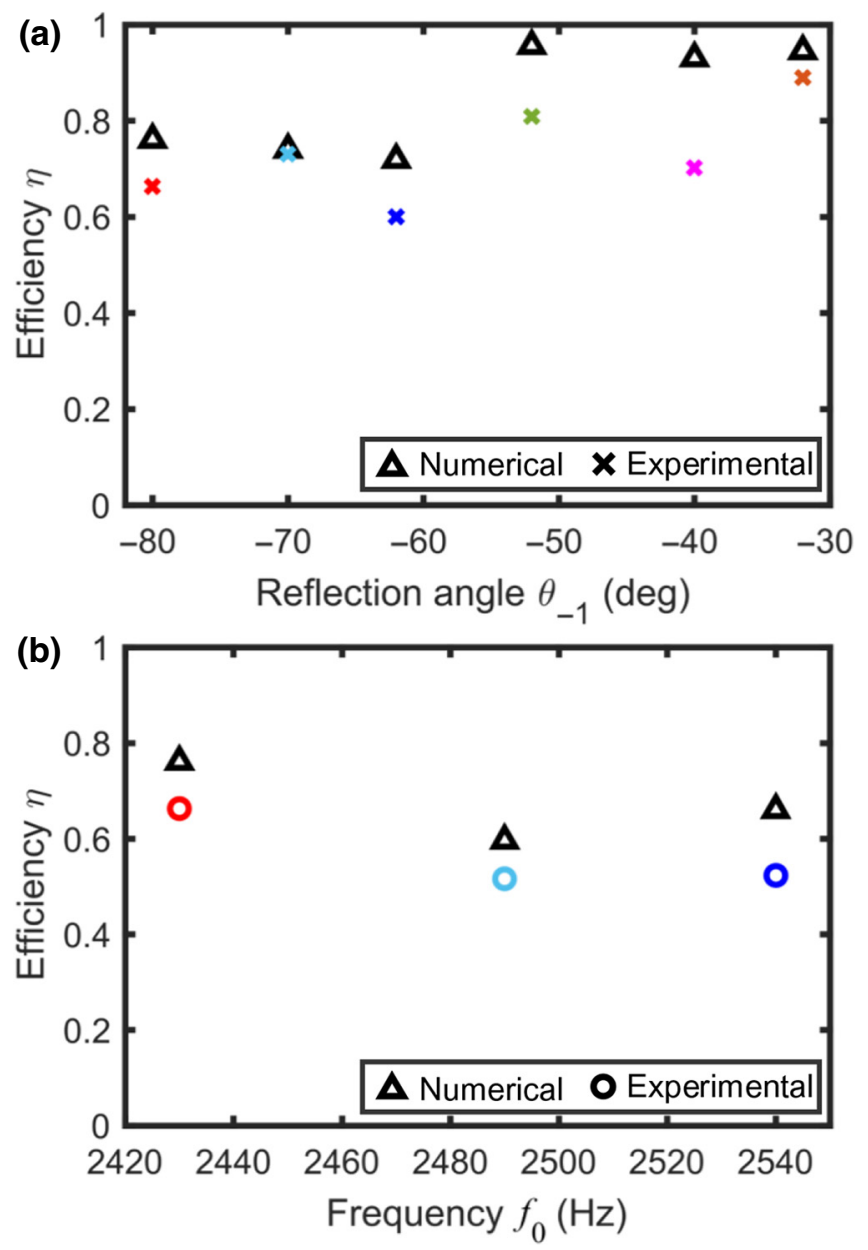

FIG. 4. Efficiency of anomalous reflection for different configurations of the acoustic metagrating. (a) Comparison between the numerical $(\triangle)$ and experimental $(\times)$ efficiency for different reflection angles $\theta_{-1}$ at $f_{0}=2430 \mathrm{~Hz}$ (see Table I for all parameters). (b) Comparison between the numerical $(\triangle)$ and experimental ( $\circ$ ) efficiency for different operating frequencies for reflection angle $\theta_{-1}=-80^{\circ}$ (see Table II for all parameters). Cross and circle markers are adopted in (a) and (b) to indicate the corresponding experimental results of the acoustic metagrating whose configurations are selected from Fig. 3 for the control of reflection angle and operating frequency, respectively. 
the experimental results of the corresponding configurations indicated in Fig. 3. The comparison between the numerically predicted $(\triangle)$ and measured $(\times)$ efficiency $\eta$, calculated from Eq. (B3), is shown in Fig. 4(a). It demonstrates that the metagrating with $\mathrm{C}$-shaped meta-atoms of fixed dimensions can reroute the incident acoustic wave into a wide range of reflection angles from $\theta_{-1}=-32^{\circ}$ to $-80^{\circ}$ by adjusting the twist angle $\beta$. Similar performance is shown in Fig. 4(b) for the reconfiguration of the operating frequency. By tuning the twist angle of scatterers as well as the corresponding period, the operating frequency is shifted while the main lobes are still directed towards $-80^{\circ}$. Full data for the angular pattern of acoustic scattering of each design are shown within the Supplemental Material [32]. We note that for angles up to $-52^{\circ}$, high efficiency can be achieved with a small number of metaatoms. However, for larger angles, a larger number of meta-atoms is required to achieve good efficiency, as discussed in Sec. II B, and Sec. 2 within the Supplemental Material [32].

\section{CONCLUSION}

A reconfigurable acoustic metagratings for highefficiency anomalous reflection, based on C-shaped metaatoms, is demonstrated numerically and experimentally. The metagrating designs presented can effectively reroute an incident acoustic wave towards extreme angles of reflection having up to $99 \%$ efficiency. The $\mathrm{C}$-shaped scatterers require less fine discretization while having reduced fabrication complexity compared to conventional gradient metasurfaces. It is also demonstrated that the metagratings can be reconfigured to achieve efficient anomalous reflection over a wide range of reflection angles and operating frequencies. This is achieved either by varying the twist angle of the meta-atoms, or by changing their distance to the backing hard wall. The demonstrated reconfigurability of this structure is particularly promising for potential applications in acoustic holography, for high-accuracy acoustic lenses and highly efficient sound barriers for noise mitigation.

\section{ACKNOWLEDGMENTS}

This research is supported by Australian Research Council Discovery Project DP200101708, and Universities Australia/DAAD joint research co-operation Scheme Project 57446203. L.Q. and A.A. are supported by the National Science Foundation.

[1] Y. Zhu, X. Fan, B. Liang, J. Cheng, and Y. Jing, Ultrathin Acoustic Metasurface-Based Schroeder Diffuser, Phys. Rev. X 7, 021034 (2017).

[2] Y. Tian, Q. Wei, Y. Cheng, and X. Liu, Acoustic holography based on composite metasurface with decoupled modulation of phase and amplitude, Appl. Phys. Lett. 110, 191901 (2017).

[3] Y. Xie, C. Shen, W. Wang, J. Li, D. Suo, B. Popa, Y. Jing, and S. Cummer, Acoustic holographic rendering with twodimensional metamaterial-based passive phased array, Sci. Rep. 6, 35437 (2016).

[4] Y. Zhu, J. Hu, X. Fan, J. Yang, B. Liang, X. Zhu, and J. Cheng, Fine manipulation of sound via lossy metamaterials with independent and arbitrary reflection amplitude and phase, Nat. Commun. 9, 1632 (2018).

[5] N. Gerard, H. Cui, C. Shen, Y. Xie, S. Cummer, X. Zheng, and Y. Jing, Fabrication and experimental demonstration of a hybrid resonant acoustic gradient index metasurface at 40 kHz, Appl. Phys. Lett. 114, 231902 (2019).

[6] J. Zhao, B. Li, Z. Chen, and C. Qiu, Manipulating acoustic wavefront by inhomogeneous impedance and steerable extraordinary reflection, Sci. Rep. 3, 2537 (2013).

[7] Y. Zhu, X. Zou, R. Li, X. Jiang, J. Tu, B. Liang, and J. Cheng, Dispersionless manipulation of reflected acoustic wavefront by subwavelength corrugated surface, Sci. Rep. 5, 10966 (2015).

[8] Y. Li, B. Liang, Z. Gu, X. Zou, and J. Cheng, Reflected wavefront manipulation based on ultrathin planar acoustic metasurfaces, Sci. Rep. 3, 2546 (2013).

[9] Y. Li, X. Jiang, R. Li, B. Liang, X. Zou, L. Yin, and J. Cheng, Experimental Realization of Full Control of Reflected Waves with Subwavelength Acoustic Metasurfaces, Phys. Rev. Appl. 2, 064002 (2014).

[10] K. Tang, C. Qiu, M. Ke, J. Lu, Y. Ye, and Z. Liu, Anomalous refraction of airborne sound through ultrathin metasurfaces, Sci. Rep. 4, 6517 (2014).

[11] Y. Li, X. Jiang, B. Liang, J. Cheng, and L. Zhang, Metascreen-Based Acoustic Passive Phased Array, Phys. Rev. Appl. 4, 024003 (2015).

[12] Y. Li, C. Shen, Y. Xie, J. Li, W. Wang, S. Cummer, and Y. Jing, Tunable Asymmetric Transmission via Lossy Acoustic Metasurfaces, Phys. Rev. Lett. 119, 035501 (2017).

[13] V. S. Asadchy, M. Albooyeh, S. N. Tcvetkova, A. DíazRubio, Y. Ra'di, and S. A. Tretyakov, Perfect control of reflection and refraction using spatially dispersive metasurfaces, Phys. Rev. B 94, 075142 (2016).

[14] N. M. Estakhri and A. Alù, Wave-Front Transformation with Gradient Metasurfaces, Phys. Rev. X 6, 041008 (2016).

[15] J. Li, C. Shen, A. Díaz-Rubio, S. A. Tretyakov, and S. Cummer, Systematic design and experimental demonstration of bianisotropic metasurfaces for scattering-free manipulation of acoustic wavefronts, Nat. Commun. 9, 1342 (2018).

[16] A. Díaz-Rubio and S. A. Tretyakov, Acoustic metasurfaces for scattering-free anomalous reflection and refraction, Phys. Rev. B 96, 125409 (2017).

[17] L. Quan and A. Alù, Passive Acoustic Metasurface with Unitary Reflection Based on Nonlocality, Phys. Rev. Appl. 11, 054077 (2019).

[18] Y. Ra'di, D. L. Sounas, and A. Alù, Metagratings: Beyond the Limits of Graded Metasurfaces for Wave Front Control, Phys. Rev. Lett. 119, 067404 (2017).

[19] H. Chalabi, Y. Ra'di, D. L. Sounas, and A. Alù, Efficient anomalous reflection through near-field interactions in metasurfaces, Phys. Rev. B 96, 075432 (2017). 
[20] V. S. Asadchy, A. Díaz-Rubio, and S. A. Tretyakov, Bianisotropic metasurfaces: Physics and applications, Nanophotonics 7, 1069 (2018).

[21] Y. Ra'di and A. Alù, Reconfigurable metagratings, ACS Photonics 5, 1779 (2018).

[22] C. F. Sieck, A. Alù, and M. R. Haberman, Origins of Willis coupling and acoustic bianisotropy in acoustic metamaterials through source-driven homogenization, Phys. Rev. B 96, 104303 (2017).

[23] M. B. Muhlestein, C. F. Sieck, P. S. Wilson, and M. R. Haberman, Experimental evidence of Willis coupling in a one-dimensional effective material element, Nat. Commun. 8, 15625 (2017).

[24] Y. Liu, Z. Liang, J. Zhu, L. Xia, O. Mondain-Monval, T. Brunet, A. Alù, and J. Li, Willis Metamaterial on a Structured Beam, Phys. Rev. X 9, 011040 (2019).

[25] L. Quan, Y. Ra'di, D. L. Sounas, and A. Alù, Maximum Willis Coupling in Acoustic Scatterers, Phys. Rev. Lett. 120, 254301 (2018).

[26] S. R. Craig, X. Su, A. Norris, and C. Shi, Experimental Realization of Acoustic Bianisotropic Gratings, Phys. Rev. Appl. 11, 061002 (2019).

[27] D. Torrent, Acoustic anomalous reflectors based on diffraction grating engineering, Phys. Rev. B 98, 060101 (2018).
[28] H. Ni, X. Fang, Z. Hou, Y. Li, and B. Assouar, Highefficiency anomalous splitter by acoustic meta-grating, Phys. Rev. B 100, 104104 (2019).

[29] Z. Hou, X. Fang, Y. Li, and B. Assouar, Highly Efficient Acoustic Metagrating with Strongly Coupled Surface Grooves, Phys. Rev. Appl. 12, 034021 (2019).

[30] A. Melnikov, Y. K. Chiang, L. Quan, S. Oberst, A. Alù, S. Marburg, and D. Powell, Acoustic meta-atom with experimentally verified maximum Willis coupling, Nat. Commun. 10, 3148 (2019).

[31] J. Jordaan, S. Punzet, A. Melnikov, A. Sanches, S. Oberst, S. Marburg, and D. Powell, Measuring monopole and dipole polarizability of acoustic meta-atoms, Appl. Phys. Lett. 113, 224102 (2018).

[32] See Supplemental Material at http://link.aps.org/supple mental/10.1103/PhysRevApplied.13.064067 for the polarizability tensor of the C-shaped scatterer, influence of number of meta-atoms and sound sources, full scattering patterns for all measured metagratings, sensitivity study of the effects of geometric parameters, two-dimensional scattered field distribution, characterization of both the infinite and finite metagratings, and the parameters of experimental structures. 